\title{
Contribution à la connaissance de la chronologie des terrasses lacustres de Banyoles (Gerona)
}

J Bech

\begin{abstract}
A brief expose of the situation and characteristics of the travertine terraces at Banyoles \{Gerona, Spain). Based on personal paleontological findings and paleoclimatic and paleopedogenic considerations, this is a contribution to the knowledge of the following travertines: Mindel for those of the " high » terrace of Usall-Espolla, Riss-Wurm for those of the "low » terrace of Banyoles-Mata and Riss for those of the possible «middle " terrace of Liô.
\end{abstract}

\section{Résumé}

Bref exposé de la situation et des caractéristiques des terrasses travertiniques de Banyoles (Gerona, Espagne). Nous basant sur des trouvailles paléontologiques personnelles et des considérations paléoclimatiques et paléopédogénétiques nous avons voulu contribuer à la connaissance de la chronologie de ses travertins : Mindel pour les travertins de la terrasse " haute " de Usall-Espoia, Riss-Wurm pour ceux de la terrasse " basse " de Banyoles-Mata et pour ceux de la terrasse « moyenne » possible de Liô.

\section{Citer ce document / Cite this document :}

Bech J. Contribution à la connaissance de la chronologie des terrasses lacustres de Banyoles (Gerona). In: Bulletin de l'Association française pour l'étude du quaternaire, vol. 8, n¹, 1971. pp. 15-20.

doi : $10.3406 / q u a t e .1971 .1167$

http://www.persee.fr/doc/quate_0004-5500_1971_num_8_1_1167

Document généré le 17/10/2015 


\title{
CONTRIBUTION A LA CONNAISSANCE DE LA CHRONOLOGIE DES TERRASSES LACUSTRES DE BANYOLES (GERONA) *
}

\author{
PAR \\ J. BECH \\ Chargé de cours à la Faculté des sciences \\ Collaborateur du Département de Géographie de l'Université de Barcelone.
}

\begin{abstract}
Résumé. - Bref exposé de la situation et des caractéristiques des terrasses travertiniques de Banyoles (Gerona, Espagne).

Nous basant sur des trouvailles paléontologiques personnelles et des considérations paléoclimatiques et paléopédogénétiques nous avons voulu contribuer da la connaissance de la chronologie de ses travertins : Mindel pour les travertins de la terrasse "haute * de Usall-Espoia, Riss-Würm pour ceux de la terrasse "basse pour ceux de la terrasse "moyenne " possible de Lio.
\end{abstract}

Abstract. - A brief exposé of the situation and characteristics of the travertine terraces at Banyoles (Gerona, Spain).

Based on personal paleontological findings and paleoclimatic and paleopedogenic considerations, this is a contribution to the knowledge of the following travertines : Mindel for those of the "high terrace of Usall-Espolla, Riss-Wurm for those of the "low" terrace of Banyoles-Mata and Riss for those of the possible "midale terrace of Lió.

\section{INTRODUCTION}

Le bassin lacustre de Banyoles (Gérona) se trouve situé à l'extrémité nord du Gironés, à la limite de la Garrotxa et de l'Ampurdan.

Le dit bassin s'est creusé dans les marnes bleues (xalió dans le pays) de l'Eocène, probablement moyen, utilisant le compartiment de la grande faille, qui suivant une direction N-S sépare le noyau montagneux de la Garrotxa de la plaine de l'Ampurdan. Cette faille, d'âge pliocène probable, coupe une grande masse de gypse, qui en se karstifiant, a préfiguré les lignes générales du bassin seulement modifié postérieurement par le remplissage des travertins et calcaires lacustres précités, par les épisodes tectoniques pléistocénes et par l'érosion récente.

Le lac actuel, dont les coordonnées sont : $42^{\circ} 7^{\prime}$ lat. $N, 2^{\circ} 47^{\prime}$ long. de Greenwich, est de type tectonokarstique et est alimenté par un limnocrène, dont le débit est de $600 \mathrm{l} / \mathrm{s}$ et la superficie approximative de $100 \mathrm{ha}$, reste de ce qui fut un grand lac pléistocène, réduit probablement à la vingtième partie de sa surface initiale.

Le témoignage de la grande étendue atteinte autrefois est constitué par d'importants dépôts de travertin qui forment les terrasses lacustres.

Traditionnellement, on a admis l'existence de deux terrasses :

1. Celle de Usall Espoia, terrasse « haute " ou de travertin " ancien ";

2. Celle de Banyoles, terrasse « basse » ou de travertin « moderne » bien qu'il soit probable qu'existe aussi une terrasse * moyenne».

* Manuscrit déposé le 11 mai 1970. 


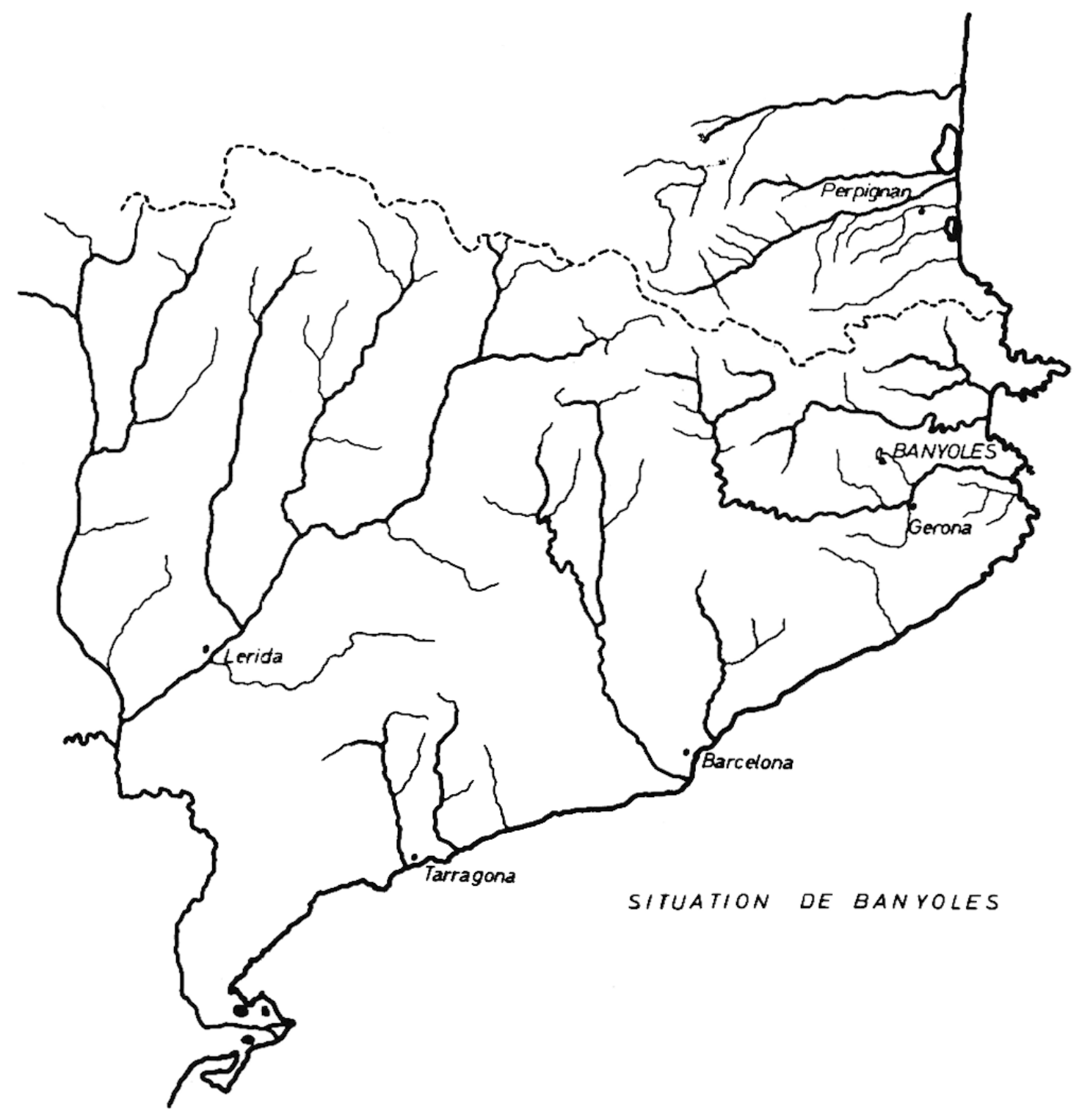

\section{TERRASSES LACUSTRES}

\section{TerRasse tRaVertinique « HaUte "}

Elle se trouve à environ $60 \mathrm{~m}$ au-dessus du niveau du lac actuel. Elle forme le plateau de Usall-Espoia-Martís, d'une surface approximative de $12 \mathrm{~km}^{2}$, situé au N-E du lac et limité au N par le Ser, affluent du Fluviá; dont le talweg le coupe sur son bord N-E. Les collines pliocènes de Santenys le limitent à l'Est.

Le travertin qui le constitue est très dur et compact, surtout vers sa partie supérieure. A sa base il est sablonneux et moins compact.

En général, il a un aspect "inorganique"; il est difficile d'apprécier des débris végétaux et animaux fossilisés. Cependant, nous avons trouvé de nombreux gastéropodes limnicoles dans sa masse, de même qu'à l'observation au microscope de lames minces nous avons vu des structures "algales" et quelques ostrácodes. 
Dans sa partie supérieure il est karstifié, avec des phénomènes d'altération qui ont produit un sol rouge, probablement terra rossa.

L'épaisseur de cette formation, selon les sondages de Vidal Pardal, est de $140 \mathrm{~m}$, au moins à Espoia, lieu qui, comme l'indique Solé Sabarís, "doit correspondre au centre de l'ancien bassin lacustre».

\section{Terrasse travertinique « Basse ».}

Entourant le lac, et à 4 à $7 \mathrm{~m}$ environ au-dessus du niveau actuel, se trouve la terrasse travertinique "basse" ou de travertin " moderne".

Elle forme la vaste plaine de Banyoles, Mata et Cornellá de Terri, d'une surface approximative de $21 \mathrm{~km}^{2}$.

Elle est constituée par un travertin poreux, tendre, feuilleté, en général stratifié, avec incrustations de Chara et autres plantes aquatiques, quelques os, plumes d'oiseaux, etc.

Cette roche sédimentaire est si tendre qu'on la scie facilement et qu'on l'exploite en carrières, l'employant pour la construction sous forme de plaques. Elle reçoit le nom populaire de turó. VIDAL et MASCARó l'ont appelé toba, la distinguant bien du travertin " ancien» de Usai, plus dense et plus dur.

Cette formation atteint une épaisseur maxima de $80 \mathrm{~m}$, dans sa partie ouest.

\section{TERRASSE " MOYENNE " POSSIBLE.}

Non citée jusqu'à présent dans la littérature scientifique.

Nous croyons que les bancs travertiniques de la zone de Lió, qui est coupée

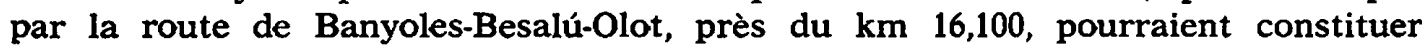
les restes d'une terrasse " moyenne" possible.

Elle est constituée par un travertin sablonneux, tendre, stratifié, à gastéropodes lacustres, avec de clairs indices de tectonisation.

Cette formation rappelle le travertin des "Estunes", toujours considéré comme équivalent de la terrasse basse de Banyoles-Mata, malgré sa hauteur supérieure au-dessus du niveau actuel du lac.

Mais, pour le moment, il n'y a pas de faits suffisants pour appuyer cette hypothèse. De plus, il ne faut pas oublier les effets que la grande faille qui traverse le lac en direction N-S a pu produire sur le travertin de la terrasse " haute " de Usall. Il pourrait aussi s'agir de blocs qui auraient glissé sous l'action de phases tectoniques supérieures au Pléistocène moyen.

\section{CHRONOLOGIE}

\section{ANTÉCÉdENTS.}

Tous les auteurs sont unanimes sur l'âge du travertin tendre, turó, de la terrasse basse de la plaine de Banyoles et sont d'accord sur le fait qu'il s'agit d'un dépôt quaternaire.

Par contre, il existe deux opinions distinctes au sujet de l'âge du travertin de la terrasse " haute" de Usall-Espoia.

Quelques auteurs, Alsius, Mascaró, Solé Sabarís et Ripoll, affirment qu'il s'agit d'une formation pléistocene, tanđis que d'autres, Mariano Vidal, Marcet, 
M. San Miguel et Vidal Pardal dans certains de ses travaux, lui attribuent un âge pliocène.

En résumé, Mariano VIDAl considère comme pliocènes lesdits travertins « pour leur vaste étendue et leur parenté avec certains conglomérats de nature tertiaire évidente $n$.

Les arguments des défenseurs de l'âge pléistocène sont les suivants :

$1^{\circ}$ La complète horizontalité dans toutes ses parties.

$2^{\circ}$ Le fait de reposer directement sur les conglomérats tertiaires. Cela est évident dans les falaises existant sur les rivières Ser et Fluviá.

A ces deux arguments stratigraphiques, s'en ajoutent deux autres, un paléontologique et l'autre géomorphologique.

3. L'existence de Hippopotamus amphibius major à ce niveau. AlsIUs trouve une canine inférieure et une molaire dans la plaine d'Espoia.

$4^{\circ}$ Solk SABarís rapproche cette terrasse haute de Usall de la terrasse de $65 \mathrm{~m}$ du Fluviá « d'âge mindélien probable \#.

\section{COUPE GEOLOG/QUE DE LA CUVETTE LACUSTRE}

DE BANYOLES (GERONA)

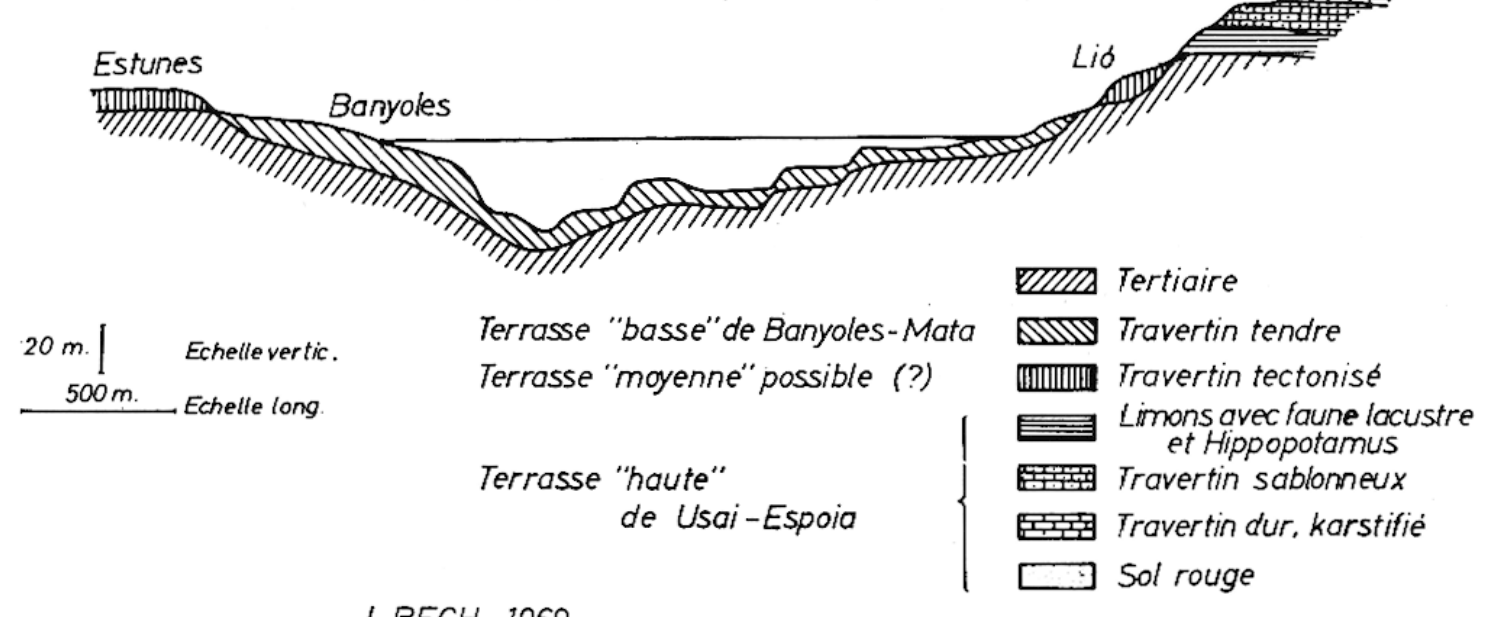

\section{J. BECH 1969}

Selon RIPOLl, on a trouvé à sa base une flore mindélienne. Ce dernier auteur affirme que le travertin d'Usall « peut s'attribuer à la fin du Mindel ou, ce qui paraît plus vraisemblable, au Mindel-Riss».

\section{Précisions sUr la terrasse « haUte ».}

Nous avons trouvé à la base du travertin d'Usall, dans les limons jaunâtres d'une carrière située au $\mathrm{km} \mathrm{16,2} \mathrm{de} \mathrm{la} \mathrm{route} \mathrm{de} \mathrm{Banyoles} \mathrm{à} \mathrm{Olot} \mathrm{par} \mathrm{Besalú,} \mathrm{quel-}$ ques ossements et une pièce buccale de Hippopotamus amphibius major que nous décrivons à part (BECH, BoRrás (J.), $1969 \mathrm{c}$ ). Nous croyons que cette découverte confirme l'âge pléistocène dudit travertin.

Et précisant davantage, nous croyons qu'on peut admettre ce qui suit:

a) Le niveau des argiles bleues à faune abondante de gastéropodes lacustres, sur lesquelles reposent les limons jaunâtres, pleins également de coquilles de 
mollusques limnicoles, dans lequel nous avons découvert Hippopotamus, peut s'attribuer à l'interglaciaire chaud Gunz-Mindel.

b) La terrasse de travertin « ancien » d'Usall, dont les matériaux reposent en concordance avec les limons bleus et jaunâtres déjà cités, doit correspondre au Mindel, en phase pluvio-glaciaire (ainsi que nous l'avons établi dans un autre travail (BECH BoRRÁs (J.), 1969 b).

c) D'autre part, en nous basant sur des considérations pédogénétiques et paléoclimatiques, nous croyons aussi très logique à l'âge mindélien de ce niveau de travertin * ancien", car le long processus de karstification du travertin et la genèse postérieure de la terra rossa qui le couvre, avec des épaisseurs pouvant atteindre plus d'un mètre, requièrent une durée de temps seulement compatible avec celle de l'interglaciaire Mindel-Riss (de 200000 années, selon PeNCK).

Précisions sur la terRasse « BasSe 》.

Le travertin tendre de la plaine de Banyoles en général a été considéré comme Würm. Ce fait est basé sur la découverte à environ $4 \mathrm{~m}$ de profondeur de la mandibule néanderthalienne et aussi de Cervus elaphus.

RIPoll pense à la possibilité d'une attribution à l'interglaciaire Riss-Würm.

Nous croyons que le dépôt de turó commence déjà au Riss-Würm. En effet nous y avons trouvé Equzds caballus (qui selon F. de VIllalta pourait être Equus stenonis cf. major Boule). Pour des raisons stratigraphiques, il peut difficilement s'agir de Equus stenonis, mais de toute manière c'est bien un Equus caballus à caractère archaïque marqué.

Etant donné le caractère archaïque de ce fossile, et étant donné que la culture moustérienne, correspondant à Homo neanderthalensis, apparaît déjà au Riss-Würm, et pensant d'autre part à la grande puissance de ce dépôt, mise en évidence par les sondages de VIDAL PaRdal, nous croyons qu'on doit attribuer ce travertin, au moins dans sa partie basale, au Riss-Würm et non au Würm comme on le faisait jusqu'à présent.

\section{INDICATION SUR LA TERRASSE « MOYENNE » POSSIBLE.}

Ayant pu démontrer l'existence de cette terrasse, nous croyons qu'elle serait d'âge rissien.

Et il se pourrait aussi, comme nous l'avons suggéré antérieurement, que le travertin des « Estunes» soit de la même époque.

Nous avons vu des symptômes de tectonisation, microplis, niveaux un peu lignitifères, gastéropodes, etc., qui rappellent beaucoup plus le travertin de Lió, possible travertin rissien ou de terrasse "moyenne", que le travertin de la terrasse basse de Banyoles-Mata ${ }^{\text {. }}$

1. J'exprime ma gratitude aux professeurs VILA VAlentf et F. de Villalta pour m'avoir fait la faveur de leur aide et de leurs conseils. 


\section{BIBLIOGRAPHIE}

Alsius (P.). - 1872-1881. Ensaig históric de la Vila de Banyoles.

Bech Borrás (J.). - 1969 b. * Genesis de travertinos lacustres : caso de Bañolas *, Revista de Geografia (sous presse).

BECH BORRAS (J.). - 1969 c. « Nuevo hallazgo de Hippopotamus amphibius major en Banyoles ", Acta Geol Hisp. (sous presse).

BENTABOL (H.). - 1910. * Hidrología superficial y subterránea de la provincia de Gerona. Estudio sobre los lagos y manantiales de Bañolas, Espolla y San Miguel de Campmajor ", Bol. de la Comisión del Mapa Geológico de España, vol. XXX, cuad. 2, 1910.

Mascaro (J.M².). - 1914. Topografía médica de Bañolas, Gerona.

Rios (J.M) y Masachs Alavedra (V.). - 1953. Bañolas (Gerona), Explicación y hoja $\mathrm{n}^{\circ} 295$ del Mapa Geológico de España.

Ripoll Perello (E.) y Lumley (H. de). - 1965. « El Paleolítico medio en Cataluña », Ampurias, XXVI-XXVII, Inst. Prehis. y Arqued. Dip. Prov. Barcelona.

San Miguel (M.) y Marcet (J.). - 1926. Guía de la excursión C4 del XIV Congreso Geoló. gico Internacional, Madrid, 1926.

Sole SABARIS (L.). - 1957. Emporda et region volcanique d'Olot, INQUA, V* Congrès International, Barcelone-Madrid, 1957.

Sole Sabaris (L.). - 1958. Geografia de Catalunya, I, Ed. Aedos, Barcelona, 1958.

VIDAL (L.). - 1908. * Investigaciones de hidrología subterranea de la comarca de Bañolas, de la provincia de Gerona », Mem. R. Ac. C. Art. Barcelona, 3" ep., v. VII, núm. 5.

Vidal Pardal (M.). - 1954. * Los yesos de la comarca de Bañolas (Gerona)», Min. Obr. Publ. Jef. Sond. Cim. c Inf. Geol. Bol., $\mathrm{n}^{\circ} 1$.

Vidal Pardal (M.). - 1960. * La alimentación subterranea del lago de Bañolas y algunos datos sobre los depositos lacustres de sus immediaciones \%, Min. Obr. Publ. Bol., $n^{\circ}$ 7, mayo 1960, Serv. Geol. Infor. y Est, p. 23-40.

Wasmund (E.). - 1934. « Der Karstquellsee Estany de Banyoles in Katalonien », Geographische Wochenschrift, vol. 2, p. 221. 